\title{
EFECTO DEL TIPO DE PROPULSION EN LA FRECUENCIA CARDIACA MAXIMA Y EN LA PERCEPCION DEL ESFUERZO EN CARRERA.
}

\author{
Pietro Scaglioni Solano y Luis Fernando Aragón-Vargas \\ Laboratorio de Rendimiento Físico y Salud (LARENFISA) \\ Escuela de Educación Física y Deportes \\ Universidad de Costa Rica, San José, Costa Rica \\ E-mail: pscaglio@cariari.ucr.ac.cr
}

\begin{abstract}
Resumen
Scaglioni, P. y Aragón-Vargas, L. F. (2001). Efecto del tipo de propulsión en la frecuencia cardiaca máxima y en la percepción del esfuerzo en carrera. Revista de Ciencias del Ejercicio y la Salud, 1(2), 25-30. Este estudio examina el efecto del tipo de propulsión sobre la Frecuencia Cardíaca (FC) y el Esfuerzo Percibido (EP, Escala de Borg) en el ejercicio de carrera, obtenidas durante dos protocolos equivalentes, en terreno plano (pendiente 0\%), uno con propulsión externa (motor, en la banda sinfín) y otro con autopropulsión (carrera en pista de atletismo). 14 corredores (34.3 +/- 9.77 años, 1.74 +/- 0.06m, 75.3 +/- $10.3 \mathrm{Kg}$ ) realizaron un protocolo que consistía en un calentamiento de 30 min. a una intensidad entre 60 y 70\% de FCmáx. seguido de un incremento gradual de la intensidad del ejercicio, aumentando la velocidad de calentamiento $1.6 \mathrm{Km} / \mathrm{h}$ cada $30 \mathrm{seg}$., llevando al sujeto a un esfuerzo máximo en un lapso de 3 a 4 minutos, se recupera hasta la condición de calentamiento, se repite dos veces el proceso de incremento gradual y recuperación, escogiendo el valor máximo de FC y EP de los tres obtenidos. En el protocolo de pista, se marcó el paso al sujeto con una bicicleta provista de un velocímetro calibrado. Se realizaron mediciones de EP durante el calentamiento (EPC) y de FC y EP durante los esfuerzos máximos (FCM, EPM). La FC del calentamiento se mantuvo constante para ambos protocolos (119.2 +/- 5.3 latidos/min; 65 +/- $2.4 \%$ de FCmáx). Se compararon los datos obtenidos con propulsión externa, (EPC1, FCM1, EPM1) y los obtenidos con autopropulsión (EPC2, FCM2, EPM2) aplicando pruebas T. No se encontraron diferencias significativas para la FCmáx entre los dos protocolos $(F C M 1=181.8+/ 8.3$; FCM2=181.5+/- 6.9). Para EP se encontraron diferencias significativas $(p<0.01)$ entre los dos protocolos para el esfuerzo máximo $(E P M 1=16.22+/-2.12 ; E P M 2=15.11+/-2.09)$ y también para la fase de calentamiento $(E P C 1=10+/-1$; EPC2 = 8.56+/- 1.74). Los resultados indican que no hay un efecto del tipo de propulsión en la FC máx en carrera, por lo que se puede utilizar indistintamente una prueba en banda sinfín o en el campo para estimar un valor máximo de FC, y que más bien lo que existe es una percepción de esfuerzo mayor en las pruebas realizadas en una banda sinfín dentro de un laboratorio tanto a niveles máximos como submáximos.

PALABRAS CLAVES: carrera, frecuencia cardiaca, percepción del esfuerzo, prueba máxima.
\end{abstract}

\section{INTRODUCCIÓN}

El término frecuencia cardíaca máxima (FCmáx) es un concepto muy utilizado por los fisiólogos del ejercicio, los atletas y los entrenadores para desarrollar una escala de valores porcentuales basados en la FCmáx para la prescripción del ejercicio (Boulay, Simoneau, Lortie y Bouchard, 1997).

Se ha utilizado la FCmáx. como un término genérico para todo tipo de actividades o disciplinas, y se han realizado investigaciones (Solano y Salazar, 1997; Iuliano 1998) donde se confirma que el valor de FCmáx difiere entre las disciplinas deportivas, como la natación, el ciclismo y el atletismo; y difiere también con el tiempo de duración de la prueba y el tipo de protocolo utilizado para su medición (Ingjer, 1991).

Las pruebas de esfuerzo máximo que se realizan en laboratorio, se llevan a cabo en banda sinfín o en cicloergómetro, y al igual que los resultados del estudio de Solano y Salazar. (1997), se demuestra que la FCmáx obtenida en la carrera es mayor que en el ciclismo.

El Colegio Americano de Medicina Deportiva (American College of Sports Medicine, ACSM, 1986), indica que una manera de estimar 
la frecuencia cardíaca máxima en forma aproximada es restándole la edad al valor hipotético de 220, que se supone es la FC máxima alcanzable (FCmáx $=220$ - edad), sin embargo la estimación de la FCmáx de esta forma indirecta puede representar un error de cerca de 10 latidos (Roitman, Pavlisko y Schulz, 1978), lo cual hace que la prescripción del ejercicio sea imprecisa.

El monitoreo de la frecuencia cardiaca (FC) y el uso de escalas de intensidad basadas en valores de FCmáx se utiliza ampliamente para controlar o verificar la intensidad de trabajo, sobre todo en el caso de atletas de deportes de resistencia (McArdle, Katch y Katch, 1986; Karvonen, y Vuormaa, 1988; Roitman y col, 1978). Obviamente, la utilidad de una escala de intensidad basada en los valores de FCmáx depende de valores de FC obtenidos con una prueba confiable de FCmáx (Edwards, 1993).

Por otro lado, el esfuerzo percibido medido a través de una escala, también es muy utilizado como criterio para determinar cuándo se ha llegado a un máximo en una prueba de esfuerzo y con más frecuencia, para determinar la intensidad del ejercicio sin tener que recurrir a métodos tradicionales como la medición de la frecuencia cardíaca (FC) o el consumo de oxígeno $\left(\mathrm{VO}_{2}\right)$. La escala de esfuerzo percibido ha sido aceptada como un método confiable para la descripción subjetiva de la intensidad del ejercicio (Cecci y Hassmen, 1991), por lo que es un parámetro de utilidad para comparar el esfuerzo autopropulsado y el ejercicio propulsado externamente.

Se han realizado estudios que demuestran diferencias en los valores de esfuerzo percibido (EP) obtenidos en una pista al aire libre y en un laboratorio (Ceci y Hassmen, 1991; Glass, Whaley, y Wegner, 1991), pero nunca se han relacionado estas diferencias con el tipo de propulsión involucrada en ningún estudio.

La práctica general para definir el valor de FCmáx es medir directamente o estimar el $\mathrm{VO}_{2}$ máx o la FCmáx de la persona a través de una prueba, o calcular el valor a través de una fórmula de estimación. Luego se asigna un programa de trabajo que incluye sesiones de diferentes niveles de intensidad que corresponden a algún porcentaje de estos valores máximos, lo que le permitirá establecer la intensidad requerida del ejercicio aeróbico durante la práctica de acuerdo a las indicaciones del entrenador (Boulay y cols., 1997).

Sin embargo, parece que no existe un método general aceptado para la obtención de FCmáx. Muchos estudios que reportan mediciones de FCmáx no describen un procedimiento concreto (Coyle, Coggam, Hopper, y Walters, 1988; Hagan, Upton, Duncan, y Gettman, 1987; Housh, Thorland, Pohnson, Hughes y Cisar, 1988; Pate, Sparling, Wilson, Cureton, y Miller, 1987; Scrimgeour, Noakes, Adams, y Myburgh, 1986; Tanaka, Nakagawa, Hazama, Matsuura, Asano, y Iseik, 1985; Boleau y cols, 1997).

A menudo, el valor dado proviene de una prueba de consumo máximo de oxígeno, donde se somete al sujeto a una prueba con incrementos graduales de carga, ya sea a través de la pendiente y la velocidad en una banda sinfín, o a través de la resistencia o "frenado" en una bicicleta ergométrica. Generalmente este tipo de pruebas tienen un breve período de calentamiento, y luego se empiezan a aumentar las cargas con intervalos de tiempo definidos, de 1 a 3 minutos, dependiendo del tipo de protocolo utilizado (Carrera, 1994).

Para banda sinfín son muy utilizados los protocolos de Balke y de Bruce, que diseñaron pruebas de esfuerzo, muy utilizadas en hospitales y clínicas, para pacientes de recuperación cardíaca o para cualquier persona que desee conocer su potencia aeróbica a través de la medición del $\mathrm{VO}_{2}$, o a través de la FCmáx para un valor dado de carga (McArdle y cols., 1986).

Para distintos tipos de atletas se han desarrollado pruebas más específicas para tener acceso a un valor de esfuerzo máximo más confiable (Brahler y Blank, 1995).

Ingjer (1991), menciona la importancia del tiempo de calentamiento, el estado de descanso del sujeto antes de la prueba, y del tiempo y número de carreras para la obtención de FCmáx. Con un protocolo de 3 carreras hasta la fatiga de 3-4 minutos cada una, luego de 30 minutos de calentamiento y con un tiempo de recuperación de 2 minutos entre carreras, en dicho estudio se obtuvieron los valores promedio más altos para FCmáx.

En cuanto al tiempo de calentamiento, Ingjer (1991) encontró que la duración de este período afecta el valor de la FCmáx, encontrándose 
valores más altos de FCmáx para una prueba con calentamiento más largo (de 2030 minutos). Los beneficios del calentamiento están relacionados con la temperatura de la superficie corporal y de los músculos, con el sistema de transporte de oxígeno y con la movilización de varias hormonas Ingjer (1979).

En el estudio de Ingjer (1991) también se comparó una prueba de laboratorio con una prueba de campo, donde se obtuvieron valores significativamente mayores para la prueba de laboratorio. En realidad el autor no da una explicación clara para estos resultados entre una prueba llevada a cabo en un laboratorio (banda sinfín) y una prueba de campo (pista de carreras o terreno similar). Una de las razones pudo ser que los protocolos de ambas pruebas no eran exactamente iguales.

Otra de las explicaciones podría estar en función del estudio del mecanismo de propulsión que impulsa al corredor durante cada tipo de prueba. No se sabe de ningún estudio que analice el efecto del tipo de propulsión que lleva al sujeto al esfuerzo máximo, como es el caso de una banda sinfín y una prueba de campo en pista. En la primera condición el sujeto es obligado a llegar a un máximo gracias a un medio propulsado externamente. La otra condición se da cuando el sujeto llega al máximo gracias a su propia impulsión, como por ejemplo en una banda sinfín sin motor o en carrera máxima sobre una pista o terreno externo.

En resumen, en un tipo de prueba el esfuerzo máximo es voluntario (autopropulsado) y en el otro caso, se puede decir que el esfuerzo máximo es obligado (propulsado externamente).

Sería importante saber si realmente existe diferencia entre una prueba llevada a cabo en banda sinfín o en el campo, ya que de existir diferencia, se debería tener cuidado a la hora de usar los resultados de una prueba o de la otra para prescribir ejercicio a cualquier atleta o persona que requiera del dato de FCmáx para cualquier otro propósito y que requiera un valor lo más objetivo y confiable posible.

Otro factor que podría estar afectando es el hecho de que se han encontrado diferencias en variables cinemáticas al comparar la carrera sobre terreno firme y sobre una banda sinfín, como por ejemplo, la diferencia en el ángulo de contacto del pie con la superficie a la hora del aterrizaje, siendo menor el ángulo en el caso de la banda sinfín, lo que podría dar una pista en cuanto a diferencias cinemáticas que afecten al desarrollo de la prueba y a una eventual diferencia en cuanto a rendimiento en una prueba máxima (Nigg, De Boer y Fischer, 1995).

El objetivo de este proyecto fue el de comparar pruebas máximas del mismo tipo de disciplina, pero bajo condiciones diferentes de propulsión del sujeto, con un protocolo similar para ambos tipos de pruebas.

El problema planteado fue : ¿Bajo qué condiciones se obtiene la FCmáx mayor y el valor más alto de EP, bajo esfuerzo voluntario (autopropulsado) o bajo esfuerzo obligado (propulsado externamente)?

La hipótesis a probar partió del supuesto que los máximos esfuerzos ( en FC y en percepción) se producen bajo condiciones de propulsión externa, partiendo de los hallazgos de la investigación de Ingjer (1991) y de acuerdo a las observaciones de Nigg y cols. (1995).

\section{METODOLOGÍA}

\section{Sujetos}

En el estudio participaron 14 Atletas experimentados en deportes de resistencia (corredores y triatlonistas) (34.3 +/- 9.77 años, $1.74+/-0.06 \mathrm{~m}, 75.3+/-10.3 \mathrm{Kg})$.

A estos se les informó acerca del tipo de prueba y los riesgos e incomodidades que pudieran surgir debido a que las pruebas máximas son agotadoras física y mentalmente.

\section{Procedimiento}

Los sujetos realizaron dos pruebas de esfuerzo máximo, separadas un mínimo de cuatro días entre sí, con pendiente del cero por ciento $(0 \%)$, y un protocolo gradual similar para ambos tipos de prueba, con el fin de que el diseño fuera lo más equivalente posible. Se aleatorizó el orden de los tratamientos.

Los valores iniciales de FCmáx se obtuvieron de datos personales suministrados por los sujetos y de estimaciones obtenidas a través de la fórmula de cálculo con la edad (FCmáx = 220 - edad). 
Para la medición del esfuerzo percibido (EP) se utilizó la escala de Borg de 20 puntos y se realizaron mediciones durante la fase de calentamiento (aproximadamente a los 15 minutos) y durante los esfuerzos máximos.

\section{Pruebas:}

1. Prueba de esfuerzo máximo con propulsión externa (en banda sinfín):

Se inicia con un calentamiento previo de 30 minutos a una intensidad aproximada al 60$70 \%$ de la FCmáx estimada. Inmediatamente, la velocidad de la banda se incrementa 1.6 $\mathrm{Km} / \mathrm{h}$ cada 30 segundos hasta un nivel que lleve al sujeto a la fatiga al final de la carrera, que en total tenía una duración de 3 a 4 minutos. Se realizaron 3 carreras de este tipo, con un intervalo de recuperación, hasta llegar a una frecuencia cardiaca dada $(60-70 \%$ FCmáx prevista).

2. Prueba de esfuerzo máximo con autopropulsión: (en pista de atletismo).

Igualmente se inicia con un calentamiento previo de 30 minutos a una intensidad aproximada al $60-70 \%$ de la FCmáx prevista. Luego, se realizan carreras alrededor de la pista, incrementando la velocidad 1.6 $\mathrm{Km} / \mathrm{h}$ cada 30 segundos, controlando el aumento progresivo de la intensidad y de la velocidad por medio de una bicicleta (equipada con un medidor de velocidad debidamente calibrado) que va delante del corredor, marcando el paso del atleta. Se realiza esto hasta un nivel que lleve al sujeto a la fatiga al final de la carrera, que en total tenía una duración de 3 a 4 minutos. Se realizaron 3 carreras de este tipo, con un intervalo de recuperación, hasta llegar a una frecuencia cardiaca dada (60-70\% FCmáx prevista).

\section{Instrmentos}

- Banda sinfín PACER ACCUMILL, 2.5 Hp.

- Pista de atletismo de aprox. $400 \mathrm{~m}$, terreno plano.

- Bicicleta con velocímetro calibrado.
- Monitor de frecuencia cardíaca POLAR ACCUREX PLUS

- Interfase POLAR PLUS

- Computador con programa POLAR TRAINING ADVISOR

\section{Análisis estadístico}

Para evaluar el efecto del tipo de propulsión sobre las variables de FC y EP, se realizaron mediciones de EP de la fase de calentamiento (EPC), a los 15 minutos de iniciado el calentamiento y de FC y EP durante cada uno de los esfuerzos máximos (FCM, EPM). Se escogió el valor más alto de FCM y de EPM para el análisis.

Dentro de la estadística descriptiva se determinaron los promedios y las desviaciones estándar de todos los sujetos en cada prueba para estos parámetros.

Para determinar el efecto de los tratamientos sobre las variables se aplicaron pruebas $t$ comparando los datos obtenidos en la prueba con propulsión externa, realizada en banda sinfín (EPC1, FCM1, EPM1) con los resultados de la prueba con autopropulsión (pista de atletismo), (EPC2, FCM2, EPM2).

\section{RESULTADOS}

En la Tabla 1 se presentan los resultados de la prueba autopropulsada y la prueba con propulsión externa.

En la Figura 1 se puede apreciar que no se encontraron diferencias significativas entre los valores de FCmáx de las dos pruebas (FCM1= $181.8 \pm 83 ;$ FCM2 $=181.5 \pm 6.99)$.

Tabla 1. Resumen de Resultados (promedios y desviaciones standard)

\begin{tabular}{ccc}
\hline Variable & $\begin{array}{c}\text { Prueba } \\
\text { propulsión } \\
\text { externa }\end{array}$ & $\begin{array}{c}\text { Prueba auto- } \\
\text { propulsada }\end{array}$ \\
\hline EPC $^{* *}$ & $10 \pm 1$ & $8.56 \pm 1.74$ \\
$\mathrm{EPM}^{* *}$ & $16.22 \pm 2.12$ & $15.11 \pm 2.09$ \\
FCM $($ lat $/ \mathrm{min})$ & $181.8 \pm 8.3$ & $181.5 \pm 6.9$ \\
\hline
\end{tabular}

$* *: \mathrm{p}<0.01$ 
Figura 1

Frecuencia Cardíaca en esfuerzo máximo

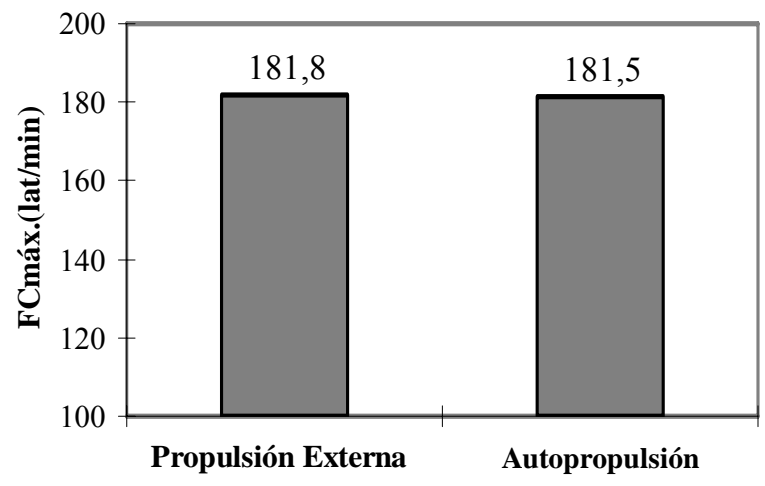

En las Figura 2 y 3, se puede apreciar que para el esfuerzo percibido EP, se encontraron diferencias significativas ( $\mathrm{p}<0.01$ ), entre las dos pruebas (propulsión externa y autopropulsión) para las dos condiciones que se midieron: la etapa de calentamiento $(\mathrm{EPC} 1=10 \pm 1 ; \mathrm{EPC} 2=8.56 \pm$ 1.74) y el esfuerzo máximo (EPM1 $=16.22 \pm$ 2.12; $\mathrm{EPM}=15.11 \pm 2.09)$.

Figura 2

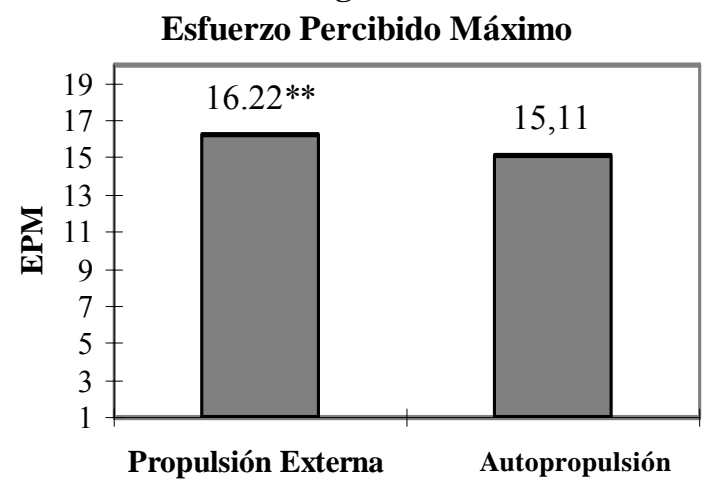

**: significativamente diferentes $(\mathrm{p}<0.01)$

Así también es importante recalcar que en el tercer intento fue donde se obtuvieron la mayoría de valores máximos para ambos tipos de prueba $(50 \%$ de las pruebas). También se observaron sujetos donde el máximo se presentó tanto en el segundo intento como en el tercer intento coincidiendo en el el mismo valor $(25 \%$ de las pruebas).
Figura 3

Esfuerzo Percibido de calentamiento

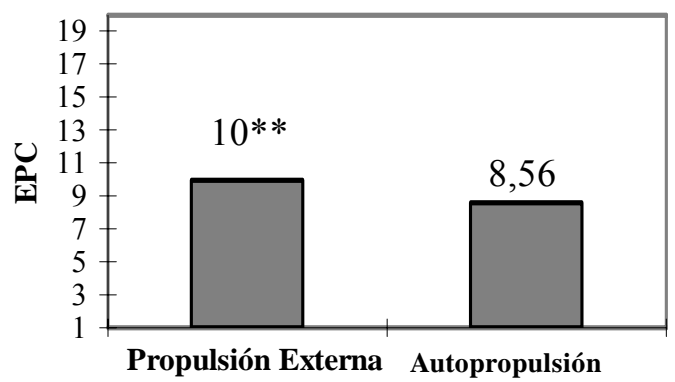

**: significativamente diferentes $(\mathrm{p}<0.01)$

\section{DISCUSION}

El propósito principal de este estudio fue el de investigar la influencia del tipo de propulsión en el ejercicio de carrera horizontal sobre las variables de FC y EP en esfuerzo máximo.

Los resultados obtenidos (presentados en la Tabla 1 y las Fig. 1, 2 y 3) muestran diferencias significativas en los niveles de EP en las dos condiciones medidas ( nivel de esfuerzo máximo y fase de calentamiento), mientras que no se hallaron diferencias en la FC durante el esfuerzo máximo para las dos pruebas (propulsión externa y autopropulsión).

Se ha sugerido que la sensación del esfuerzo durante la carrera al aire libre (carrera autopropulsada) puede ser reducida por otras percepciones que interfieren tales como la sensación de la resistencia del viento, termorregulación a través del viento, entradas visuales como paisajes, sol, montañas, movimiento, etc. (Potteiger y Weber, 1994).

Las diferencias encontradas en la percepción del esfuerzo, además de estar relacionadas con el tipo de propulsión, podrían verse afectadas por el "efecto del ambiente", ya que hay mecanismos perceptuales, como el procesamiento de información externa vs. pistas internas, que afectan el proceso de percepción durante las pruebas. (Pennebaker y Lightner, 1980). Lo anterior también podría estar relacionado con el estrés debido a la falta de costumbre de ejercitarse sobre una banda sinfín dentro de una habitación cerrada, ya que los sujetos están acostumbrados a 
realizar ejercicio al aire libre (Ceci y Hassmen, 1991).

Los resultados en cuanto a FCmáx indican que en ésta no hay un efecto del tipo de propulsión, por lo que se puede utilizar indistintamente una prueba en banda sinfín o una de campo para estimar un valor máximo de FC, y que más bien lo que existe es una percepción de esfuerzo mayor en las pruebas realizadas en una banda sinfín dentro de un laboratorio, tanto en ejercicio de baja intensidad (65\% FCmáx) como en esfuerzo máximo, contrario a los resultados del estudio de Ingjer (1991). Esta contradicción con dicho estudio puede deberse a que el autor no utilizó protocolos exactamente iguales para la prueba de campo y la de laboratorio, lo que sí se tomó en cuenta en el presente estudio.

De acuerdo a los resultados de este estudio, el efecto de las diferencias cinemáticas en cuanto a la carrera sobre terreno firme y sobre una banda sinfín señaladas por Nigg y cols (1995) parecen no afectar en el caso de esfuerzos máximos en cuanto al parámetro de $\mathrm{FC}$.

Por otro lado, se corrobora la importancia de utilizar un protocolo con 3 "picos" de esfuerzo, ya que la mitad de los valores máximos más altos del presente estudio se obtuvieron en el tercer intento y un $25 \%$ en el segundo y tercer intento. Esto refuerza los resultados obtenidos por Ingjer (1991), donde se menciona que el cuerpo necesita un volumen de trabajo de consideración para poder alcanzar la FCmáx, sobre todo si son atletas de resistencia.

\section{REFERENCIAS}

American College of Sport Medicine (1986). Guidelines for exercise testing and prescription. $3^{\text {rd }}$ ed. Philadelphia, USA: Lea \& Febiger.

Boulay, MR., Simoneau, JA., Lortie G. \& Bouchard C. (1997). Monitoring high-intensity endurance exercise with heart rate and tresholds; Med. Sci. Sports Exerc. 29 (1), 125-132.

Brahler, CJ., and Blank, SE. (1995). Versaclimbing elicits higher VO2máx than does treadmill running or rowing ergometry. Med. Sci. Sports Exerc. 27(2), 249-254.

Carrera, JL. (1994). Economía del corredor. Selección. 3 (2): 96-101.

Ceci, R. and Hassmen, P. (1991). Self-monitored exercise at three different RPE intensities in treadmill vs field running. Med. Sci. Sports Exerc. 26(9), 791-796.
Coyle, EF., Coggam, AR., Hopper, MK. \& Walters, TJ. (1988). Determinants of endurance in well trained cyclists. J Appl. Physiol. 64: 2622-2630.

Edwards S. (1993). The Heart Rate Monitor Book. (4 ed). NY, Polar CIC INC.

Glass, S.C., Whaley, MH. \& Wegner, MS. (1991). Ratings of perceived exertion among standard treadmill protocols and steady state running. Int. J. of Sports Med. 12:7782.

Hagan, RD., Upton, SJ., Duncan, JJ. \& Gettman, LR. (1987). Marathon performance in relation to maximal aerobic power training indices in female distance runners. Br J Sports Med, 21: 3-7.

Housh, TJ., Thorland, WG., Pohnson, GO., Hughes, RA. \& Cisar, CJ. (1988). The contribution of selected physiological variables to middle distance running performance. J Sports Med, 28: 20-26.

Ingjer F. (1991). Factors influencing assesment of maximal heart rate. Scand J Med Sci Sports. 1: 134-140.

Ingjer, F. and Stromme, S.B. (1979). Effects of active, passive and no warm-up on the physiological response to heavy exercise. Eur. J. Appl. Physiol. 40: 273-282.

Iuliano, S., Naughton, G., Collier, G. \& Carlson, J. (1998). Examination of the self-selected Fluid Intake Practices by Junior Athletes During a Simulated Duathlon Event. Int J Sport Nutr, 8: 10-23.

Karvonen, J. and Vuormaa, T. (1988). Heart rate and exercise intensity during sports activities. Practical aplicaation. Sports Med: 5 : 303-312.

McArdle, WD. Katch, FI. \& Katch, VI. (1986). Fisiología del Ejercicio. Madrid: Alianza Editorial S. A.

Nigg, BM., De Boer, RW. \& Fischer, V. (1995). A kinematic comparison of overground and treadmill running. Med. Sci. Sports Exerc. 27(1), 98-105.

Pate, RR., Sparling, PB., Wilson, GE., Cureton, KJ. \& Miller, BJ. (1987). Cardiorespiratory and metabolic responses to submaximal and maximal exercise in elite women distance runners. Int J Sports Med, 8: 91-95.

Pennbaker, JW., and Lightner. (1980). Competition of internal and external information in an exercise setting. J. Pers. Soc. Psychol. 39: 165-174.

Potteiger, JA. and Weber, SF. (1994). Rating of perceived exertion and heart rate as indicators of exercise intensity in different environmental temperatures. Med. Sci. Sports Exerc. 23 (6) 732-738.

Roitman, J., Pavlisko, JJ. \& Schulz, GW. (1978). Exercise prescription by heart rate methods. Phys Sportsmed 6:98-102.

Scrimgeour, AG., Noakes, TD., Adams, B. \& Myburgh, K. (1986). The influence of weekly training distance on fractional utilization of maximum aerobic capacity in marathon and ultramaathon runners. Eur $J$ Appl Physiol. 55:202-209.

Solano, R. and Salazar, W. (1997). Frecuencias cardíacas máximas en las disciplinas del triatlón. V Simposio Internacional de Ciencias del Deprote y la Salud. Universidad de Costa Rica. (Sin publicar)

Tanaka, K., Nakagawa, T., Hazama, T., Matsuura, Y., Asano, K. \& Iseik, TA. (1985). Prediction equation for indirect assesment of anaerobic threshold in male distance runners. Eur J Appl Physiol. 57: 203-209. 